\section{(2) OPEN ACCESS}

\begin{abstract}
- Prepublication history for this paper is available online. To view these files, please visit the journal online (http://dx. doi.org/10.1136/thoraxjnl2018-212812).
\end{abstract}

For numbered affiliations see end of article.

Correspondence to Dr Sam M Janes, Lungs for Living Research Centre, UCL Respiratory, University College London, London, UK; s.janes@ucl.ac.uk

Received 8 November 2018 Revised 16 July 2019 Accepted 7 August 2019 Published Online First 26 September 2019

\section{SLinked}

- http://dx.doi.org/10.1136/ thoraxjnl-2019-213790

Check for updates

(c) Author(s) (or their employer(s)) 2019. Re-use permitted under CC BY. Published by BMJ.

To cite: Ruparel M, Quaife SL, Dickson JL, et al.

Thorax 2019;74:1140-1146.

\title{
Evaluation of cardiovascular risk in a lung cancer screening cohort
}

\author{
Mamta Ruparel (D) ,' Samantha L Quaife, ${ }^{2}$ Jennifer L Dickson, ${ }^{1}$ Carolyn Horst, ${ }^{1}$ \\ Stephen Burke, ${ }^{3}$ Magali Taylor, ${ }^{4}$ Asia Ahmed, ${ }^{4}$ Penny Shaw, ${ }^{4}$ May-Jan Soo, ${ }^{3}$ Arjun Nair, ${ }^{4}$ \\ Anand Devaraj, ${ }^{5}$ Emma Louise O'Dowd (D) , ${ }^{6}$ Angshu Bhowmik, ${ }^{7}$ Neal Navani, ${ }^{1,8}$ \\ Karen Sennett, ${ }^{9}$ Stephen W Duffy, ${ }^{10}$ David R Baldwin, ${ }^{6}$ Reecha Sofat, ${ }^{11}$ Riyaz S Patel, ${ }^{11}$ \\ Aroon Hingorani, ${ }^{11}$ Sam $M$ Janes
}

\section{ABSTRACT}

Introduction Lung cancer screening (LCS) by low-dose computed tomography (LDCT) offers an opportunity to impact both lung cancer and coronary heart disease mortality through detection of coronary artery calcification (CAC). Here, we explore the value of CAC and cardiovascular disease (CVD) risk assessment in LCS participants in the Lung Screen Uptake Trial (LSUT). Methods In this cross-sectional study, current and ex-smokers aged 60-75 were invited to a 'lung health check'. Data collection included a CVD risk assessment enabling estimation of 10 year CVD risk using the QRISK2 score. Participants meeting the required lung cancer risk underwent an ungated, non-contrast LDCT. Descriptive data, bivariate associations and a multivariate analysis of predictors of statin use are presented. Results of 1005 individuals enrolled, 680 were included in the final analysis. 421 (61.9\%) had CAC present and in $49(7.2 \%)$, this was heavy. $668(98 \%)$ of participants had a QRISK2 $\geq 10 \%$ and QRISK2 was positively associated with increasing $C A C$ grade (OR 4.29 (CI 0.93 to 19.88 ) for QRISK2 $=10 \%-20 \%$ and 12.29 (CI 2.68 to 56.1 ) for QRISK2 $220 \%$ respectively). Of those who qualified for statin primary prevention (QRISK2 $\geq 10 \%), 56.8 \%$ did not report a history of statin use. In the multivariate analysis statin use was associated with age, body mass index and history of hypertension and diabetes.

Conclusions LCS offers an important opportunity for instituting CVD risk assessment in all LCS participants irrespective of the presence of LDCT-detected CAC. Further studies are needed to determine whether CAC could enhance uptake and adherence to primary preventative strategies.

\section{INTRODUCTION}

In the National Lung Screening Trial (NLST), annual lung cancer screening (LCS) by low-dose computed tomography (LDCT), improved lung cancerspecific mortality by $20 \%$ and all-cause mortality by $6.7 \%$ compared with annual chest $\mathrm{x}$-ray. ${ }^{1}$ The benefit in lung cancer-specific mortality has since been confirmed by the Dutch-Belgian Lung Cancer Screening Trial (NELSON) ${ }^{2}$ and the Multicentric Italian Lung Detection (MILD) investigators. ${ }^{3}$ Unsurprisingly, following the reduction in lung cancer death in the LDCT arm in NLST, and given the age and

\section{Key messages}

What is the key question?

- What is the prevalence of coronary artery calcium, cardiovascular risk and statin use as primary prevention against cardiovascular disease among a cohort of individuals undergoing lung cancer screening?

What is the bottom line?

- Coronary artery calcification was present in $61.9 \%$ of lung cancer screening participants and was positively associated with cardiovascular disease risk; $98 \%$ qualified for a statin, though less than half reported using one.

Why read on?

- These findings highlight the opportunity for influencing cardiovascular disease outcomes through lung cancer screening and suggest that cardiovascular disease risk assessment should be considered for inclusion in lung cancer screening programmes.

smoking history of the cohort, coronary heart disease (CHD) was responsible for the majority of total deaths in the LDCT arm. ${ }^{1}$ Globally, CHD accounts for the greatest number of deaths annually, ${ }^{4}$ and given both lung cancer and CHD risk are associated with increasing age and smoking history, the LCS population is at disproportionately high risk of CHD-related morbidity and mortality.

In this context, there may be an important opportunity for LCS and LDCT to also help reduce CHD mortality, through simultaneous assessment of cardiovascular disease (CVD) risk, via quantification of coronary calcium, a marker of established atherosclerosis. The coronary artery calcium (CAC) or Agatston score, correlates highly with the volume and burden of plaque seen at post mortem and shows an association with incident CHD risk, such that a score of 1000 has been associated with a 10-fold increased risk of all-cause mortality. ${ }^{5}$ In contrast, a CAC score of 0 is associated with low risk, similar to the background population. ${ }^{6-10}$

While CAC is visible on LDCT, differences do exist compared with dedicated cardiac scanning, in 
protocols and in particular, the absence of electrocardiography (ECG) gating. Non-gated images are subject to motion artefact and run the risk of inadequately estimating the CAC burden. Nevertheless, several studies have shown that CAC assessments on non-gated LDCT scans are comparable to formal ECG-gated CAC measurements ${ }^{11-14}$ with good agreement between the methods. Direct comparison between these methods has demonstrated underestimation of high CAC in $0 \%-23.4 \%$ of cases, ${ }^{15}$ while overestimation of CAC may also occur. However, these discrepancies may not be of clinical significance if undetected CAC in this group does not translate into cardiovascular events, and the high-risk individuals are appropriately managed. Further studies assessing visual CAC scoring in LCS have shown it to be a reliable predictor of cardiovascular events. Importantly, very low event rates have been reported by non-gated LDCT scans in the $\mathrm{CAC}=0$ group, suggesting there may be a utility for visual CAC reporting in the LCS population, although the definitions of 'event' and median follow-up durations of these studies were variable. ${ }^{16-21}$

Quantifying CVD risk through assessment of CAC alone is not recommended, ${ }^{22}$ though a recent consensus document advocates reporting CAC on all non-contrast CT chest scans..$^{23}$ Addition of CAC assessment to the Framingham score can refine risk stratification, which may have a particularly meaningful impact on those in the 'intermediate' CVD risk group, ${ }^{6}$ though whether this is also true for individuals eligible for LCS is not known. In the UK, the National Institute for Health and Care Excellence (NICE) guidelines advocate assessment of cardiovascular risk using the QRISK2 algorithm. Those deemed to be at high risk, currently defined as having a $10 \%$ or greater risk for events over the next 10 years, irrespective of total cholesterol level, should be offered a statin (atorvastatin $20 \mathrm{mg}$ ) as part of a wider discussion around lifestyle interventions to reduce their risk. ${ }^{24}$

In the present study, we aimed to explore the value of coronary calcium and cardiovascular risk in asymptomatic LCS participants who did not report a prior history of CHD. In particular, we aimed to determine (1) the prevalence and extent of coronary calcium in LCS participants using a quantification system considered to be an acceptable alternative to Agatston scoring; (2) participant risk estimates using QRISK2 and how this is distributed across CAC scores and, finally, (3) the prevalence and predictors of statin use among those with both high and low risk estimates and CAC burden.

\section{METHODS}

\section{Study design, participants and setting}

This cross-sectional study is nested within the Lung Screen Uptake Trial (LSUT), the methods for which have been described previously. ${ }^{25}$ Briefly, individuals aged between 60 and 75 , who had been coded in their primary care health record as current smokers within the past 5-7 years, were invited by their primary care physician for a 'lung health check' (LHC) at one of two London hospitals between November 2015 and July 2017. The primary aim of LSUT was to test differences in uptake to LCS between individuals randomly allocated to either 'standard' invitation materials or targeted materials, designed to engage socioeconomically deprived smokers. Individuals attending the LHC were invited to participate in the study.

Those meeting the US Preventative Services Task Force (USPSTF) criteria for LCS (ie, $\geq 30$ pack-years and quit $\leq 15$ years ago), ${ }^{26}$ or a lung cancer risk of $1.51 \%$ as determined by the Prostate Lung Colorectal Ovarian study $\left(\mathrm{PLCO}_{\mathrm{m} 2012}\right)$ model $^{27}$ or $2.5 \%$ as determined by the Liverpool Lung Project (LLP) model, ${ }^{28}$ were offered a LDCT scan to screen for lung cancer. Participants were excluded from the LDCT if they did not have capacity to give consent, their weight exceeded restrictions for scanner $(>200 \mathrm{~kg})$, they were unable to lie flat, had poor physical fitness such that radical treatment would be contraindicated, or had had a CT scan of their chest within the previous 12 months. Participants were given written information on the potential benefits and harms of LCS and following a discussion with the research nurse or clinical trials practitioner, were asked to give informed consent to have an LDCT as part of LCS.

Very brief smoking cessation advice (a standardised intervention from the UK's National Centre for Smoking Cessation and Training) ${ }^{29}$ was given to all current smokers at the LHC, and participants were also randomised to receive details of their local National Health Service (NHS) smoking cessation service or be proactively referred to the smoking cessation service.

\section{Data collection}

Data were prospectively collected by a study practitioner at the LHC. Self-reported demographics (age, sex, ethnicity, education level, Index of Multiple Deprivation (IMD) score and rank), smoking status and history, cardiovascular and lung cancer risk factors (including all those contained in the QRISK2, PLCO $_{\mathrm{m} 2012}$ and LLP models), history of CHD and number of general practice (GP) attendances in the past year were recorded. Family history was defined as per the QRISK2 model and was assessed as 'angina or heart attack in a in a first degree relative aged $<60$ years old'. Hand-held spirometry, height, weight and blood pressure were also recorded.

\section{LDCT acquisition}

Participants undertook the examination via a 16-channel or higher multidetector, non-ECG-voltage-gated CT without the administration of intravenous contrast. Imaging was performed during suspended maximal inspiration. The lung parenchyma (lung apices to bases) was scanned in its entirety in a single craniocaudal acquisition. The field of view selected as the smallest diameter as measured from widest point of outer rib to outer rib large enough to accommodate the entire lung parenchyma. Thin detector collimation $(0.5$ $\mathrm{mm}$ ) was used. Images were reconstructed at $0.5-1.0 \mathrm{~mm}$ section thickness using standard soft tissue and lung algorithms. Radiation exposures were as low as possible while maintaining good image quality (median $1.2 \mathrm{mSv}$, IQR $0.9 \mathrm{mSv}, 1.7 \mathrm{mSv}$ ). The tube potential and tube current-time product varied according to participant body habitus and were between 80 and $120 \mathrm{kVp}$ and between 20 and $80 \mathrm{mAs}$, respectively.

\section{Outcome measures}

QRISK2 scores were calculated by ClinRisk Ltd using their QRISK2-2017 Java batch processor and used self-reported history of included risk factors. These are estimated QRISK2 scores as we did not have serum cholesterol values for participants as part of the study, and the batch processor substitutes an age-sex-ethnicityestimate of cholesterol/ high-density lipoprotein ratio when this is presented as missing. Three patients had missing systolic blood pressure values, hence the batch processor substituted an age-sexethnicity estimate of systolic blood pressure for these participants. Self-reported use of statins was recorded.

The LDCT scans were single-read by a team of five radiologists with expertise in thoracic CT reporting and experience ranging from 5 to 28 years. Reports included recording of a visual grading of coronary calcium, which was developed and validated in LCS LDCT examinations by Chiles et al. ${ }^{21}$ Here, 


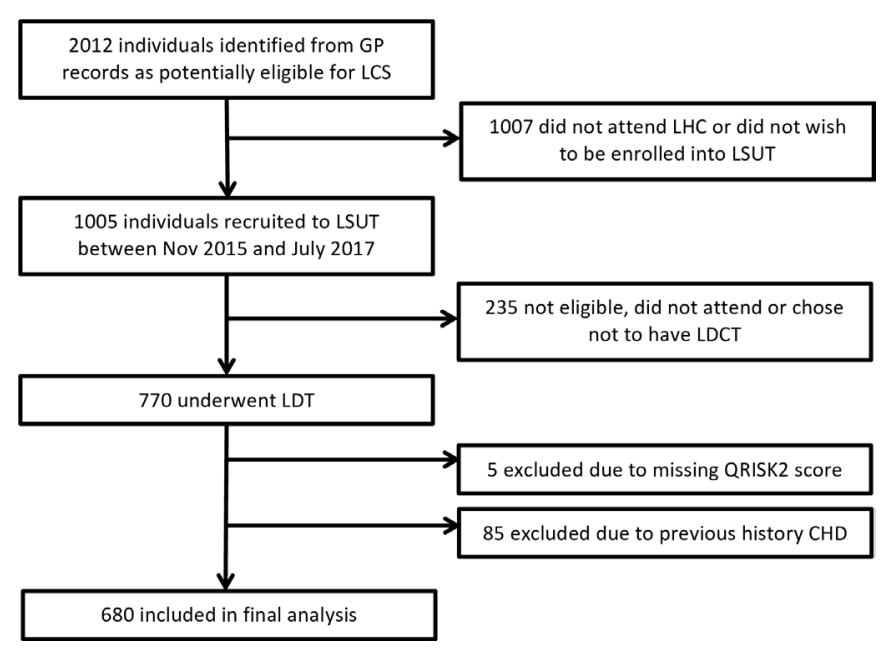

Figure 1 Flow diagram for study participants. CHD, coronary heart disease; LCS, lung cancer screening; LDCT, low-dose computed tomography; LHC, lung health check; LSUT, Lung Screen Uptake Trial.

radiologists performed a simple, overall visual assessment, on a per scan basis taking into account an 'average' of all coronary arteries. The grades of none, mild, moderate or heavy used have demonstrated good correlation with formal Agatston scores at cut offs of $0,1-100,100-1000$ and $>1000$.

\section{Sample size and statistical analysis}

The sample size of the LSUT cohort was based on the primary behavioural research question and has been described in the published protocol. ${ }^{25}$ However, retrospectively, one might reasonably have posited a priori that around two thirds of our sample would have QRISK2 score greater than 20\% and one third less than or equal to $20 \%$. One might also reasonably hypothesise that $15 \%$ of those with QRISK2 $\leq 20 \%$ would have visual CAC grade of moderate to heavy compared with a figure of $25 \%$ in those with QRISK2 > 20\%. For 80\% power to observe this difference as significant (5\% significance level, two-sided testing), we would require 621 subjects (207 with QRISK2 $\leq 20 \%$ and 414 with QRISK2>20\%). In the event, we had 680 subjects, 204 with QRISK2 $\leq 20 \%$ and 476 with QRISK2 > 20\%.

Participants who self-reported a prior history of angina, angioplasty, myocardial infarction and coronary artery bypass grafting were considered to have a prior history of $\mathrm{CHD}$ and were excluded from the analysis, as were those without an LDCT or with missing QRISK2 scores. Individuals were categorised by QRISK2 into categories of low (0\%-10\%), moderate (10\%$20 \%)$ and high risk $(\geq 20 \%)$ of CVD. Descriptive statistics were used to determine the demographic and clinical characteristics of individuals in each QRISK2 category. The distributions of QRISK2 scores by CAC grade and prevalence of CAC by grade in each QRISK2 category were summarised and compared. Associations between QRISK2 score and CAC grade were assessed using $\chi^{2}$ and multivariate ordinal logistic regression analyses, and differences in QRISK2 scores between CAC categories were evaluated using the Kruskal-Wallis test, with subsequent pairwise comparisons performed using a posthoc Dunn's test with a Bonferroni correction for multiple comparisons. Next, the prevalence of self-reported statin use was compared by QRISK2 category and bivariate associations between statin use and various clinical and demographic variables were assessed using $\chi^{2}$ analysis. A multivariate logistic regression model was used to assess independence in these associations, adjusting for age, gender, smoking status, history of hypertension, history of diabetes and body mass index (BMI). Variables for the final model were selected on the basis of clinical and statistical significance. So, those variables that were significant in univariable analysis were tested and included in the multivariable model, alongside those variables that were deemed to be a priori confounders and variables that were felt to be of important clinical significance.

Agreement between radiologists was assessed for the 5\% of LDCT scans that were second-read as part of the quality assurance process, using the weighted kappa $\left(\kappa_{\mathrm{w}}\right)$ test with quadratic weights. Missing values were excluded from the analyses (and were present for only one variable, IMD rank). Likelihood ratio testing was used for tests of significance, and $\mathrm{p} \leq 0.05$ were considered significant. Analyses were carried out using STATA V.14.

\section{RESULTS}

Out of 2012 potentially eligible individuals identified in the primary care records of 16 GP practices, 1005 attended for an LHC and were recruited into the study. Of those, 770 underwent a baseline LDCT examination. Total 85 participants were excluded due to a history of self-reported CHD and five due to missing QRISK2 score data, leaving a total of 680 participants in the final analysis (figure 1).

Participant characteristics by QRISK2 category are described in table 1 though the low numbers in the lowest QRISK2 group should be considered. $38.4 \%$ of the cohort were female (though 12 out of 12 of the QRISK $2<10 \%$ group were female) and $82.4 \%$ were white. More than half of the overall cohort left school at or before the age of 16, though the lower QRISK2 categories tended to be better educated. $88.1 \%$ of participants were from the two most socioeconomically deprived quintiles, and this distribution was similar across the three QRISK2 categories. $72.1 \%$ of participants were current smokers though as expected, this proportion was higher in the highest QRISK2 category. Smoking intensity and duration and degree of airway obstruction were also higher in the highest QRISK2 category. Similarly, participant BMI and blood pressure also increased by QRISK2 category. Figure 2 shows examples of participants with coronary calcification of mild, moderate and heavy categories.

Out of the 680 participants, 421 (61.9\%) had CAC present on LDCT. CAC grade was 'moderate' in 145 (21.3\%) and 'heavy' in $49(7.2 \%)$ (table 2). CAC grade was associated with QRISK2 category $(\mathrm{p}<0.01)$. Overall $98 \%$ of participants had a QRISK2 score of $\geq 10 \%$ and were therefore eligible for statin primary prevention. Conversely, more than half $(54.7 \%)$ of the participants with a QRISK2 of 10\%-20\% had no CAC visible.

The range of QRISK2 scores within each CAC group was wide. Median QRISK2 scores increased significantly with increasing CAC grade, $\mathrm{H}(3)=70.428, \mathrm{p}<0.01$. Adjusted $\mathrm{p}$ values for multiple pairwise comparisons of median QRISK2 scores between CAC groups were all significant except between the 'moderate' and 'heavy' CAC grades (figure 3). This association was supported by increasing ORs for each increasing QRISK2 category (table 3).

Self-reported statin use was associated with QRISK2 $(\mathrm{p}<0.01)$ and number of GP visits in the past year in the univariate analysis (table 4). Of those that did qualify for a statin based on their QRISK2, 56.8\% did not report a history of statin use, with this number being even higher (76.6\%) in the 10\%-20\% QRISK2 category. $90 \%$ of participants who were not taking statins reported visiting their GP $\geq 1$ times in the past year. In the multivariate analysis, statin use was independently associated with age, history of 
Table 1 Participant characteristics by QRISK2 category (\% totals may not sum up due to rounding)

\begin{tabular}{|c|c|c|c|c|}
\hline \multirow[t]{2}{*}{ Variables } & \multicolumn{3}{|c|}{ QRISK2 Score category: $n(\%)$ or median (IQR)* } & \multirow[b]{2}{*}{ Total } \\
\hline & $<10 \%, \mathrm{n}=12$ & $10 \%-20 \%, n=192$ & $>20 \%, n=476$ & \\
\hline Age & $62(61,63)$ & $63(62,66)$ & $67(64,70)$ & $65(63,69)$ \\
\hline Female (vs male) & $12(100)$ & $71(37.0)$ & $178(37.4)$ & $261(38.4)$ \\
\hline \multicolumn{5}{|l|}{ Ethnicity } \\
\hline White & $11(91.7)$ & $137(71.4)$ & $412(86.6)$ & $560(82.4)$ \\
\hline $\begin{array}{l}\text { Black (African or } \\
\text { Caribbean) }\end{array}$ & $0(0)$ & $32(16.7)$ & $41(8.6)$ & $73(10.7)$ \\
\hline Other & $1(8.3)$ & $23(12.0)$ & $23(4.4)$ & $47(6.9)$ \\
\hline \multicolumn{5}{|c|}{ Highest level of education } \\
\hline $\begin{array}{l}\text { Left school at or } \\
\text { before age } 15\end{array}$ & $5(41.7)$ & $76(39.6)$ & $276(58.0)$ & $357(52.5)$ \\
\hline $\begin{array}{l}\text { CSEs, O-levels or } \\
\text { equivalent }\end{array}$ & $2(16.7)$ & $22(11.5)$ & $41(8.6)$ & $65(9.6)$ \\
\hline $\begin{array}{l}\text { A-levels or } \\
\text { equivalent }\end{array}$ & $1(8.3)$ & $24(12.5)$ & $42(8.8)$ & $67(9.9)$ \\
\hline $\begin{array}{l}\text { Further } \\
\text { education }\end{array}$ & $0(0)$ & $14(7.3)$ & $19(4.0)$ & $33(4.9)$ \\
\hline Bachelor degree & $3(25.0)$ & $26(13.5)$ & $52(10.9)$ & 81 (11.9) \\
\hline $\begin{array}{l}\text { Further higher } \\
\text { degree }\end{array}$ & $1(8.3)$ & $23(12.0)$ & $40(8.4)$ & $64(9.4)$ \\
\hline \multicolumn{5}{|l|}{ IMD quintile } \\
\hline 1 (most deprived) & $5(41.7)$ & $88(45.8)$ & $276(58.0)$ & $369(54.3)$ \\
\hline 2 & $5(41.7)$ & $78(40.62)$ & $147(30.1)$ & $230(33.8)$ \\
\hline 3 & $0(0)$ & $3(1.56)$ & $13(2.7)$ & $16(2.4)$ \\
\hline 4 & $0(0)$ & $0(0)$ & $1(0.2)$ & $1(0.2)$ \\
\hline 5 (least deprived) & $0(0)$ & $0(0)$ & $0(0)$ & $0(0)$ \\
\hline \multicolumn{5}{|l|}{ Smoking status } \\
\hline $\begin{array}{l}\text { Current smoker } \\
\text { (vs former) }\end{array}$ & $2(16.7)$ & $112(58.4)$ & $376(79.0)$ & $490(72.1)$ \\
\hline $\begin{array}{l}\text { Years smoked } \\
\text { (years) }\end{array}$ & $45(42-27)$ & $45(43,48)$ & $49(45,52)$ & $47(44,51)$ \\
\hline $\begin{array}{l}\text { Average smoking } \\
\text { intensity (cigs/ } \\
\text { day) }\end{array}$ & $10(7-18)$ & $15(10,20)$ & $20(12,20)$ & $20(10,20)$ \\
\hline \multicolumn{5}{|l|}{ Lung function } \\
\hline $\begin{array}{l}\text { FEV1 (\% } \\
\text { predicted) }\end{array}$ & $89(78.5,106)$ & $84(69,100)$ & $81(63,94)$ & $81(66,96)$ \\
\hline FEV/FVC (\%) & $66(62,77)$ & $70(65,76)$ & $67(60,74)$ & $68(61,75)$ \\
\hline \multicolumn{5}{|c|}{ Other cardiovascular risk factors } \\
\hline $\begin{array}{l}\text { On hypertensive } \\
\text { treatment }\end{array}$ & $0(0)$ & $34(17.7)$ & $196(41.2)$ & $230(33.8)$ \\
\hline $\begin{array}{l}\text { Family history of } \\
\text { heart disease }\end{array}$ & $1(8.3)$ & $54(28.12)$ & $233(49.0)$ & $288(42.4)$ \\
\hline $\begin{array}{l}\text { History of } \\
\text { diabetes }\end{array}$ & $0(0)$ & $0(0)$ & $83(17.4)$ & $83(12.2)$ \\
\hline BMI $\left(\mathrm{kg} / \mathrm{m}^{2}\right)$ & $\begin{array}{l}22.9 \\
(19.8,30.2)\end{array}$ & $\begin{array}{l}25.5 \\
(22.1,28.6)\end{array}$ & $\begin{array}{l}26.1 \\
(23.1-29.3)\end{array}$ & $\begin{array}{l}25.9 \\
(22.8,29.2)\end{array}$ \\
\hline $\begin{array}{l}\text { Systolic BP (mm } \\
\mathrm{Hg})\end{array}$ & $\begin{array}{l}114 \\
(100,121)\end{array}$ & $\begin{array}{l}129 \\
(117,138)\end{array}$ & $\begin{array}{l}139 \\
(128,152)\end{array}$ & $\begin{array}{l}135 \\
(125,148)\end{array}$ \\
\hline $\begin{array}{l}\text { Diastolic BP (mm } \\
\mathrm{Hg})\end{array}$ & $73(70,75)$ & $82(75,87)$ & $84(77,91)$ & $83(76,90)$ \\
\hline
\end{tabular}

hypertension, diabetes and BMI (but not number of GP visits in the past year) (table 5). Several variables including gender, IMD score and quintile, education level, smoking status, years smoked, years quit, pack-years and systolic blood pressure were not found to be associated with statin use in the univariate analysis or after adjusting for other variables. Gender and smoking status were included in the final model as they were felt to be important a priori confounders with regards to development of CAC.

Inter-observer agreement for the double-read LDCT scans was very good according to Landis $\& \operatorname{Koch}^{30}(\kappa w=0.88, \mathrm{p}=0<0.01)$.

\section{DISCUSSION}

In this prospective observational study in a cohort of individuals undergoing an LDCT examination for LCS, we have found that $62 \%$ of participants have coronary calcium present. Second, increasing QRISK2 was associated with increasing CAC grade on LDCT. Third, $98 \%$ of LCS-eligible individuals met the $\geq 10 \%$ 10 -year CVD risk threshold required for statin primary prevention of CVD events in the UK, although less than half reported a history of statin use. In the adjusted analyses, statin use was associated with factors related to increasing cardiovascular risk, but not with frequency of prior GP visits within the past year. These data add to the debate on the role LDCT could play for enhancing parallel CVD prevention efforts as part of wider LCS programmes nationally and personalising risk management strategies.

Our data first confirm previous reports that LCS-eligible individuals have evidence of coronary disease. ${ }^{18} 21$ A recent study reporting 10-year outcomes in a subcohort of participants who met the USPSTF criteria ${ }^{26}$ for LCS from the Multi-Ethnic Study of Atherosclerosis (MESA) noted an almost threefold increase in the cardiovascular event rate $(20.8 \%)$ in this group ${ }^{31}$ than the 10 -year event rate reported in the overall MESA cohort $(7.8 \%) .{ }^{32}$ This finding highlights the potentially sizeable impact implementation of primary prevention in LCS-eligible individuals could have.

Our findings of a positive association between CAC grade and QRISK2 are in keeping with prior studies. ${ }^{33}$ Ultimately, whether CAC grade offers any clinical utility over and above the QRISK2 score for risk prediction remains a key question as most patients by virtue of age and smoking would be considered at high risk. In the context of LDCT, it is of note that a large number $(54.7 \%)$ of participants in the moderate QRISK2 category (10\%-20\%)

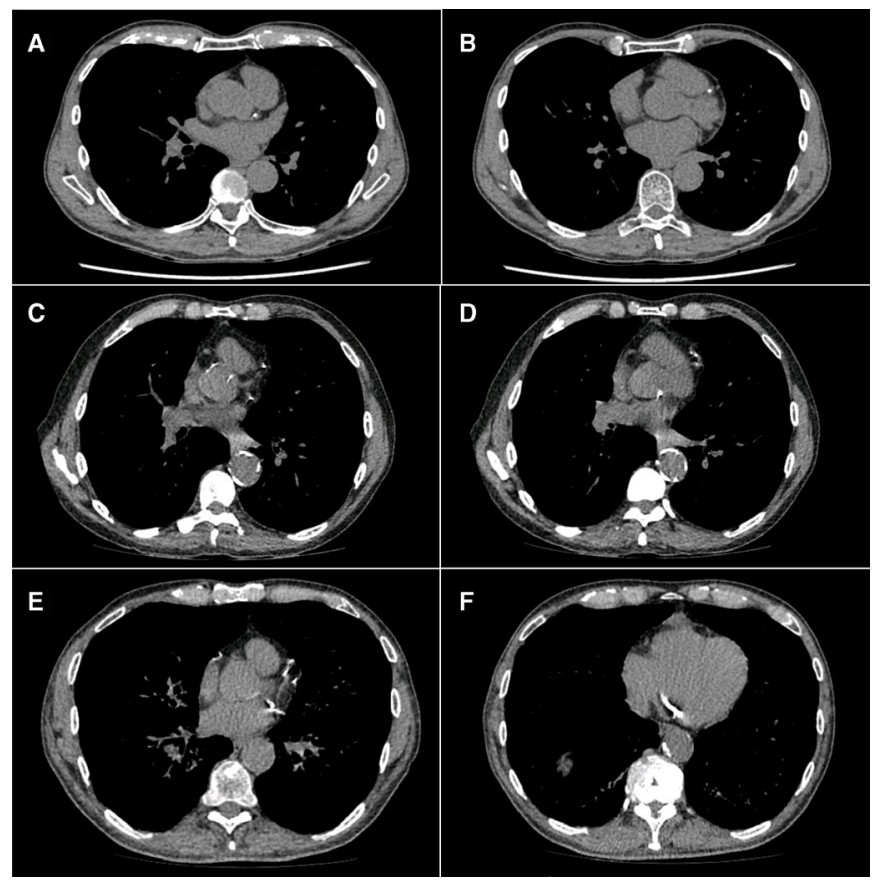

Figure 2 Examples of participants with mild $(A, B)$, moderate $(C, D)$ and heavy $(E, F)$ coronary artery calcification. 
Table 2 Prevalence of CAC on LDCT by QRISK2 score category

\begin{tabular}{|c|c|c|c|c|c|c|}
\hline \multirow[b]{2}{*}{ QRISK2 risk category } & \multicolumn{4}{|c|}{ Visual CAC grade, $n(\%)$} & \multirow[b]{2}{*}{ Total } & \multirow[t]{2}{*}{$P$ value } \\
\hline & None & Mild & Moderate & Heavy & & \\
\hline$<10 \%$ & $10(83.3)$ & $2(16.7)$ & $0(0)$ & $0(0)$ & $12(1.8)$ & $<0.01$ \\
\hline $10 \%-20 \%$ & $105(54.7)$ & $58(30.2)$ & $24(12.5)$ & $5(2.6)$ & $192(28.2)$ & \\
\hline$>20 \%$ & $144(30.3)$ & $167(35.1)$ & $121(25.4)$ & $44(9.2)$ & $476(70.0)$ & \\
\hline
\end{tabular}

CAC, coronary artery calcification; LDCT, low-dose computed tomography.

had no CAC. It has been suggested from the LCS cohorts that only a small number will have a CVD event. ${ }^{16-21}$ However, many of these studies only looked at fatal CHD events or had limited follow-up duration. More recent data from the USPSTF-eligible cohort in MESA discussed above ${ }^{31}$ demonstrated the 10 -year CVD event rate (including fatal and non fatal coronary events or stroke) to be $14.2 \%$ in this group and may be explained by the presence of non-calcific atherosclerotic disease. The wide range of QRISK2 scores in those with no CAC in the present study also supports the notion that this group cannot be assumed to have a benign outcome, though the fact that women or certain ethnic groups may have clinical risk overestimated should also be considered. Increasingly, data are emerging that combining CVD risk scoring with clinical risk factors plus CAC may offer a much more refined approach to risk stratification with greater net reclassification, especially of individuals in the intermediate risk categories. ${ }^{6}{ }^{34}$ However, in the context of LDCT, outcome studies with CAC would be needed to demonstrate the added value of risk scoring using CAC versus QRISK2 alone.

Nonetheless, $98 \%$ of LCS participants were at greater than $10 \%$ risk by QRISK2, the threshold currently recommended in the UK at which statin therapy can be considered. This proportion may be higher than expected, based on results from other LCS screening studies; however, those cohorts have typically been slightly younger and have included fewer current

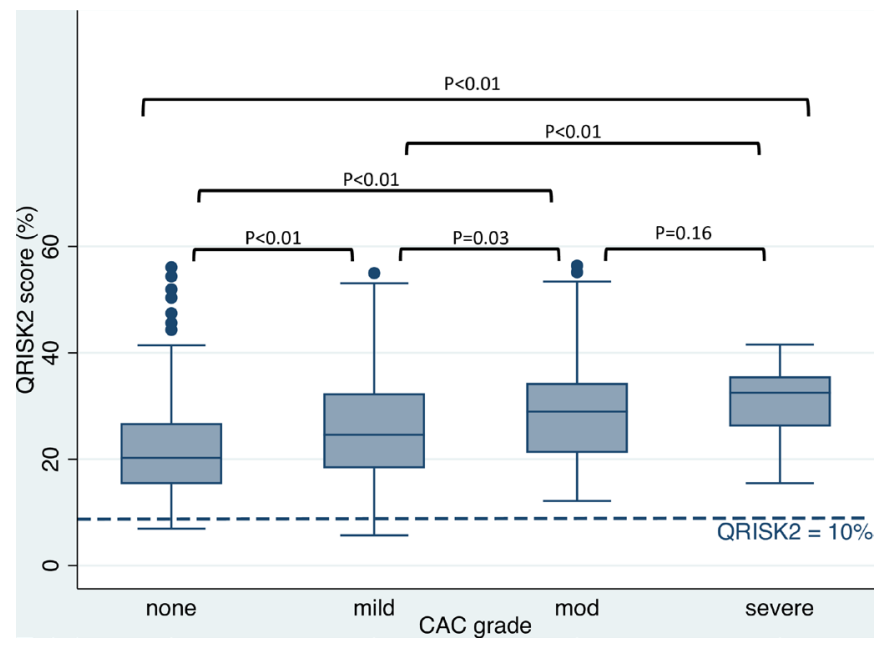

Figure 3 QRISK2 score distribution for each visually determined CAC grade on LDCT. The boxes contain the 25th to 75th QRISK2 scores within each category, with the median value represented by a solid line running through the box. The whiskers extend to the upper and lower adjacent values and the dots represent the outliers. An extra horizontal dotted line has been added to demonstrate the threshold for initiation of statin therapy for primary prevention in the UK (10\%). Posthoc $p$ values corrected for multiple comparisons between group medians are highlighted. CAC, coronary artery calcification; LDCT, low-dose computed tomography. smokers. ${ }^{135-37}$ In another recent report of another 'real-world' UK-based CT screening pilot, a similarly high QRISK2 scores were observed. ${ }^{38}$ Despite this, our analysis found that only approximately 50\% who qualified, reported statin use, falling to $23 \%$ in the $10 \%-20 \%$ QRISK2 category. This result is consistent with other UK and US studies, who have reported $46.0 \%$ and $49.7 \%$ statin use in their eligible cohorts, respectively. ${ }^{39}$ In the UK, individuals aged between 40 and 74 are invited to NHS health checks in order to carry out CVD risk assessment, though uptake to this has been low at only $30 \%$ in $2012 .^{40}$

Overall, these findings support the use of LDCT and LCS as another opportunity to engage high-risk patients for primary prevention of CVD. While it remains uncertain if CAC can improve risk stratification over and above standard risk estimation, reporting CAC using the visual grading method used in the present study is quick and may be motivational in discussions with patients and for initiating and adherence to statin and antihypertensive therapy. Certainly a systematic review has demonstrated that CAC screening improved medication adherence and could likely motivate further behavioural changes. ${ }^{41} \mathrm{~A}$ large body of research ${ }^{42}$ implicates patient-related, behavioural barriers in non-adherence to statins (eg, concerns about side effects, misconceptions about causality and symptoms, low perceived benefit) and has shown that behavioural interventions targeting these barriers are effective. ${ }^{43}$ In the UK NHS health checks, initiation of statin treatment only occurs in 20\% of NHS health check attendees with $\geq 20 \%$ CVD risk. ${ }^{40}$ Awareness of the presence of CAC may, therefore, have a positive effect on statin use in this group, particularly if barriers among those who previously declined treatment could be identified and addressed. Furthermore, as novel preventative therapies emerge, the uniformly high CVD risk in this group $(65.7 \%$ with $\geq 20 \% 10$ year risk) may warrant more discerning risk stratification strategies for which knowledge of CAC may be informative.

The current study has some limitations. First, CAC was graded visually, and not using formal Agatston scoring, however, this is an accepted and validated method ${ }^{21}$ and interobserver agreement in this study for $5 \%$ of scans that were double read was very good. The lack of cardiovascular event data limited our ability to examine if CAC adds any additional clinical utility to standard risk scores. Larger planned studies with longer follow-up will address this in due course. The inclusion criteria in the present study targeted socioeconomically deprived smokers aged $60-75,{ }^{25}$ but we feel

Table 3 Unadjusted ordinal logistic regression for association between LDCT CAC grade and QRISK2 category

\begin{tabular}{lcc}
\hline QRISK2 category & OR $(\mathrm{Cl})$ (unadjusted) & P value \\
\hline$<10 \%$ & 1.0 & \\
$10 \%-20 \%$ & $4.29(0.93-19.88)$ & 0.062 \\
$\geq 20 \%$ & $12.29(2.69-56.1)$ & $<0.01$ \\
\hline
\end{tabular}

CAC, coronary artery calcification; LDCT, low-dose computed tomography. 
Table 4 Number of individuals qualifying for a statin based on QRISK2 alone compared with self-reported history of statin use, and with self-reported number of GP attendances in the past year

\begin{tabular}{|c|c|c|c|c|}
\hline & On statin & Not on statin & $P$ value & $\begin{array}{l}\text { Not on statin, when } \\
\text { indicated by QRISK2 } \\
\text { score }(\%)\end{array}$ \\
\hline \multicolumn{5}{|c|}{ QRISK2 score category, $n$ (\% of row) } \\
\hline$<10 \%$ & $0(0)$ & $12(100)$ & $<0.01$ & 0 \\
\hline $10 \%-20 \%$ & $45(23.4)$ & $147(76.6)$ & & 76.6 \\
\hline$>20 \%$ & $237(49.8)$ & $239(50.2)$ & & 50.2 \\
\hline Total & 282 & 386 & & 56.8 \\
\hline \multicolumn{5}{|c|}{ Number of GP attendances in past year, $\mathrm{n}$ (\% of column) } \\
\hline 0 & $10(3.6)$ & $47(11.8)$ & $<0.01$ & \\
\hline $1-5$ & $211(74.8)$ & $285(71.8)$ & & \\
\hline$>5$ & $61(21.6)$ & 66 (16.4) & & \\
\hline
\end{tabular}

results are still generalisable, given emerging evidence advocating selection of LCS-eligible individuals based on lung cancer risk. ${ }^{44} 45$ For screening populations including younger participants, it should be considered that the distribution of cardiovascular risk may be lower than that observed in this study. We did not collect data on NHS health check attendance; on reasons for the lack of statin use if prescribed or if discussions had taken place about statins at all. Finally, we did not measure serum cholesterol and so our QRISK2 score used a substitute value as well as self-reported history of risk factors, which may make the scores less accurate. Measuring serum cholesterol may not add more value given the high risk of

Table 5 Univariable and multivariable logistic regression model for history of self-reported statin use

\begin{tabular}{|c|c|c|c|c|}
\hline & $\begin{array}{l}\text { OR }(\mathrm{CI}) \\
\text { (unadjusted) }\end{array}$ & $P$ value & OR (CI) (adjusted) & $P$ value \\
\hline \multicolumn{5}{|l|}{ Age } \\
\hline $60-63$ & 1 & $<0.01$ & 1 & $<0.01$ \\
\hline $64-67$ & $1.29(0.88$ to 1.90$)$ & & 1.51 (0.98 to 2.35$)$ & \\
\hline $68-71$ & 2.08 (1.36 to 3.18$)$ & & 2.36 (1.45 to 3.84$)$ & \\
\hline $72-76$ & 2.20 (1.32 to 3.66$)$ & & 2.42 (1.35 to 4.31$)$ & \\
\hline \multicolumn{5}{|l|}{ Gender } \\
\hline Male & 1 & 0.27 & 1 & 0.12 \\
\hline Female & $1.19(0.87$ to 1.61$)$ & & 1.31 (0.93 to 1.87$)$ & \\
\hline \multicolumn{5}{|c|}{ Smoking status } \\
\hline Former & 1 & 0.11 & 1 & 0.14 \\
\hline Current & $0.76(0.54$ to 1.06$)$ & & $0.75(0.51$ to 1.10$)$ & \\
\hline \multicolumn{5}{|c|}{ History of hypertension } \\
\hline No & 1 & $<0.01$ & 1 & $<0.01$ \\
\hline Yes & 4.26 (3.03 to 5.96$)$ & & 3.51 (2.44 to 5.06$)$ & \\
\hline \multicolumn{5}{|c|}{ History of diabetes } \\
\hline No & 1 & $<0.01$ & 1 & $<0.01$ \\
\hline Yes & 13.55 (6.85 to 26.8$)$ & & 11.10 (5.44 to 22.6) & \\
\hline \multicolumn{5}{|l|}{ BMI } \\
\hline$<18.5$ & $0.91(0.27$ to 3.02$)$ & $<0.01$ & $1.65(0.47$ to 5.80$)$ & 0.023 \\
\hline $18.5-25$ & 1 & & 1 & \\
\hline$>25$ & 1.64 (1.14 to 2.36$)$ & & 1.64 (1.14 to 2.36$)$ & \\
\hline
\end{tabular}

BMI, body mass index. participants due to other (smoking and age) risk factors and adds expense and time. Despite these limitations, our study was carried out in a practical and pragmatic manner with high uptake rates, making it generalisable to a population and real world setting.

\section{CONCLUSION}

Patients undergoing LDCT, as part of an LCS programme, have a high prevalence of coronary disease estimated through visual scoring of CAC and are also at high CVD risk according to standard clinical calculators. However, despite this, a substantial proportion of patients are not on statin therapy for primary prevention, despite eligibility based on 10 year CVD risk scores. We propose that LCS programmes may offer a unique opportunity to engage patients for CVD prevention by (1) helping address the underutilisation of statins in this high risk group and (2) potentially using anatomical evidence of CAC as motivation to enhance adherence to preventative interventions.

\section{Author affiliations}

${ }^{1}$ Lungs for Living Research Centre, UCL Respiratory, University College London, London, UK

${ }^{2}$ Research Department of Behavioural Science and Health, University College London, London, UK

${ }^{3}$ Department of Radiology, Homerton University Hospital NHS Foundation Trust, London, UK

${ }^{4}$ Department of Radiology, University College London Hospitals NHS Foundation Trust, London, UK

${ }^{5}$ Department of Radiology, Royal Brompton Hospital, London, UK ${ }^{6}$ Respiratory Medicine Unit, David Evans Research Centre, Nottingham University Hospitals NHS Trust, Nottingham, UK

${ }^{7}$ Respiratory Medicine, Homerton University Hospital NHS Foundation Trust, London, UK

${ }^{8}$ Thoracic Department, University College London Hospitals NHS Foundation Trust, London, UK

${ }^{9}$ Killick Street Health Centre, London, UK

${ }^{10}$ Wolfson Institute of Preventive Medicine, Barts and London, London, UK

${ }^{11}$ Institute of Cardiovascular Science, University College London, London, UK

Acknowledgements We would like to acknowledge the substantial intellectual contribution made by Professor Jane Wardle who sadly passed away prior to publication and is deeply missed by all of her coauthors, colleagues and students. We would also like to thank ClinRIsk Ltd for calculating QRISK2 scores for our cohort and to the entire Lung screen Uptake Trial team.

Contributors MR, SLQ, JLD, MT, AA, PS, SB, M-JS, AN, AD, AB, NN, DRB, SWD and $S M J$ all contributed to the design and/or of the conduct study. AN, CH, EOD, RSP, RS and $\mathrm{AH}$ made a significant intellectual contribution with interpretation of the data from the study. All authors contributed to the preparation and approved the final version of the manuscript.

Funding This study was part of the Lung Screen Uptake Trial, which was funded by a National Awareness and Early Diagnosis Initiative (NAEDI) project grant awarded by Cancer Research UK (CRUK) and a consortium of funders (Department of Health (England); Economic and Social Research Council; Health and Social Care R\&D Division, Public Health Agency, Northern Ireland; National Institute for Social Care and Health Research, Wales; Scottish Government) (SLQ and SMJ). MR, SLQ and SMJ have received funding from the Roy Castle Lung Cancer Foundation. SMJ is a Wellcome Trust Senior Fellow in Clinical Science (WT107963AIA). SMJ is supported by the Rosetrees Trust, the Stoneygate Trust, the Welton Trust, the Garfield Weston Trust and UCLH Charitable Foundation. This work was undertaken at UCLH/UCL who received a proportion of funding from the Department of Health's NIHR Biomedical Research Centre's funding scheme (NN, SMJ). SLQ is supported by a Cancer Research UK Postdoctoral Fellowship ((C50664/A24460). AN receives a proportion of funding from the Department of Health NIHR Biomedical Research Centres Funding Scheme at University College London Hospitals (UCLH)/University College London (UCL).

Competing interests SMJ, MR, JLD and $\mathrm{CH}$ are supported by funding for a large trial of low dose CT screening, called the 'SUMMIT Study' by GRAIL Inc. SQ collaborates on the SUMMIT study. SMJ has received honoraria from Astra Zeneca, BARD1 Bioscience and Achilles Therapeutics for being an Advisory Board Expert and travel to a US conference. SMJ receives grant funding from Owlstone for a separate research study and has a family member with a financial association with Astra Zeneca. MR has received travel funding for a conference from Takeda and an honorarium for speaking at educational meeting from Astra Zeneca. AN is a member of the Advisory Board for Aidence Artificial Intelligence. 
RS has received honoraria, consulting and speaker fees from Amgen, Sanofi and Bayer. SMJ, MR, JLD, CH, SQ, AN and RS perceive that these disclosures pose no academic conflict for this study. All other authors have no other competing interests to declare.

\section{Patient consent for publication Not required.}

Ethics approval This study is part of the Lung Screen Uptake Trial (LSUT), which was granted ethical approval by the City Road and Hampstead NHS Research Ethics Committee (REC; reference: 15/LO/1186) and was registered with clinicaltrials.gov (NCT02558101) and the International Standard Registered Clinical/soCial sTudy Number (ISRCTN21774741)

Provenance and peer review Not commissioned; externally peer reviewed. Data availability statement Data are available on reasonable request.

Open access This is an open access article distributed in accordance with the Creative Commons Attribution 4.0 Unported (CC BY 4.0) license, which permits others to copy, redistribute, remix, transform and build upon this work for any purpose, provided the original work is properly cited, a link to the licence is given, and indication of whether changes were made. See: https://creativecommons.org/ licenses/by/4.0/.

\section{ORCID iDs}

Mamta Ruparel http://orcid.org/0000-0001-8880-6567

Emma Louise O'Dowd http://orcid.org/0000-0001-6904-1327

Sam M Janes http://orcid.org/0000-0002-6634-5939

\section{REFERENCES}

1 Aberle DR, Adams AM, Berg CD, et al. Reduced lung-cancer mortality with low-dose computed tomographic screening. N Eng/ J Med 2011;365:1-15.

2 De Koning H, Van Der Aalst C, Ten Haaf K, Koning HJD, Carlijn M, Aalst VD, et al. PL02.05 effects of volume CT lung cancer screening: mortality results of the Nelson Randomised-Controlled population based trial. Journal of Thoracic Oncology 2018;13.

3 Pastorino U, Silva M, Sestini S, et al. Prolonged lung cancer screening reduced 10-year mortality in the mild trial: new confirmation of lung cancer screening efficacy. Ann Oncol.

4 WHO. Top 10 causes of death, 2018. Available: http://www.who.int/gho/mortality_ burden_disease/causes_death/top_10/en/ [Accessed 5 Oct 2018].

5 Budoff MJ, Shaw LJ, Liu ST, et al. Long-Term prognosis associated with coronary calcification: observations from a Registry of 25,253 patients. J Am Coll Cardiol 2007;49:1860-70.

6 Greenland P, LaBree L, Azen SP, et al. Coronary artery calcium score combined with Framingham score for risk prediction in asymptomatic individuals. JAMA 2004;291.

7 Bonow RO. Clinical practice. should coronary calcium screening be used in cardiovascular prevention strategies? N Engl J Med 2009;361:990-7.

8 Valenti V, Ó Hartaigh B, Heo R, et al. A 15-year Warranty period for asymptomatic individuals without coronary artery calcium: a prospective follow-up of 9,715 individuals. JACC Cardiovasc Imaging 2015;8:900-9.

9 Nasir K, Bittencourt MS, Blaha MJ, et al. Implications of coronary artery calcium testing among statin candidates according to American College of Cardiology/ American heart association cholesterol management guidelines: MESA (multi-ethnic study of atherosclerosis). J Am Coll Cardiol 2015;66:1657-68.

10 Mortensen MB, Falk E, Li D, et al. Statin Trials, Cardiovascular Events, and Coronary Artery Calcification. JACC Cardiovasc Imaging 2018;11:221-30.

11 Budoff MJ, Nasir K, Kinney GL, et al. Coronary artery and thoracic calcium on noncontrast thoracic CT scans: comparison of ungated and gated examinations in patients from the COPD gene cohort. J Cardiovasc Comput Tomogr 2011;5:113-8.

12 Einstein AJ, Johnson LL, Bokhari S, et al. Agreement of visual estimation of coronary artery calcium from low-dose CT attenuation correction scans in hybrid PET/CT and SPECT/CT with standard Agatston score. J Am Coll Cardiol 2010;56:1914-21.

13 Kim SM, Chung MJ, Lee KS, et al. Coronary calcium screening using low-dose lung cancer screening: effectiveness of MDCT with retrospective reconstruction. AJR Am J Roentgenol 2008;190:917-22.

14 MT W, Yang P, Huang YL, et al. Coronary arterial calcification on low-dose ungated MDCT for lung cancer screening: concordance study with dedicated cardiac CT. Am J Roentgenol 2008:190:923-8.

15 Xie X, Zhao Y, de Bock GH, et al. Validation and prognosis of coronary artery calcium scoring in nontriggered thoracic computed tomography: systematic review and metaanalysis. Circ Cardiovasc Imaging 2013;6:514-21.

16 Shemesh J, Henschke $\mathrm{Cl}$, Shaham $\mathrm{D}$, et al. Ordinal scoring of coronary artery calcifications on low-dose CT scans of the chest is predictive of death from cardiovascular disease. Radiology 2010;257:541-8.

17 Sverzellati N, Cademartiri F, Bravi F, et al. Relationship and prognostic value of modified coronary artery calcium score, FEV1, and emphysema in lung cancer screening population: the mild trial. Radiology 2012;262:460-7.

18 Jacobs PC, Gondrie MJA, van der Graaf Y, et al. Coronary artery calcium can predict all-cause mortality and cardiovascular events on low-dose CT screening for lung cancer. AJR Am J Roentgenol 2012;198:505-11.
19 Takx RAP, Išgum I, Willemink MJ, et al. Quantification of coronary artery calcium in nongated CT to predict cardiovascular events in male lung cancer screening participants: results of the Nelson study. J Cardiovasc Comput Tomogr 2015;9:50-7.

20 Rasmussen T, Køber L, Abdulla J, et al. Coronary artery calcification detected in lung cancer screening predicts cardiovascular death. Scand Cardiovasc J 2015;49:159-67.

21 Chiles C, Duan F, Gladish GW, et al. Association of coronary artery calcification and mortality in the National lung screening trial: a comparison of three scoring methods. Radiology 2015;276:82-90.

22 Montalescot G, Sechtem U, Achenbach S, et al. 2013 ESC guidelines on the management of stable coronary artery disease: the task force on the management of stable coronary artery disease of the European Society of cardiology. Eur Heart J 2013:34:2949-3003.

23 Hecht HS, Cronin P, Blaha MJ, et al. 2016 SCCT/STR guidelines for coronary artery calcium scoring of noncontrast noncardiac chest CT scans: a report of the Society of cardiovascular computed tomography and society of thoracic radiology. J Cardiovasc Comput Tomogr 2017;11:74-84.

24 NICE. Cardiovascular disease: risk assessment and reduction, including lipid modification, 2014. https://www.nice.org.uk/guidance/cg181/chapter/4-Otherinformation

25 Quaife SL, Ruparel M, Beeken RJ, et al. The Lung Screen Uptake Trial (LSUT): protocol for a randomised controlled demonstration lung cancer screening pilot testing a targeted invitation strategy for high risk and 'hard-to-reach' patients. BMC Cancer 2016;16:281.

26 U.S. Preventive services Task force. U.S. preventive services Task force: final recommendation statement, 2013. Available: http://www.uspreventiveservicestask force.org/uspstf13/lungcan/lungcanfinalrs.htm\#summary [Accessed 28 Nov 2014].

27 Tammemägi MC, Katki HA, Hocking WG, et al. Selection criteria for lung-cancer screening. N Engl J Med 2013;368:728-36.

28 Cassidy A, Myles JP, van Tongeren M, et al. The LLP risk model: an individual risk prediction model for lung cancer. Br J Cancer 2008;98:270-6.

29 National Centre for Smoking Cessation and Training (NCSCT). Very brief advice training module. Available: http://www.ncsct.co.uk/publication_very-brief-advice.php [Accessed 9 Jul 2018].

30 Landis JR, Koch GG. The measurement of observer agreement for categorical data. Biometrics 1977:33:159-74

31 Leigh A, McEvoy JW, Garg P, et al. Coronary Artery Calcium Scores and Atherosclerotic Cardiovascular Disease Risk Stratification in Smokers. JACC Cardiovasc Imaging 2019;12:852-61

32 Budoff MJ, Young R, Burke G, et al. Ten-Year association of coronary artery calcium with atherosclerotic cardiovascular disease (ASCVD) events: the multi-ethnic study of atherosclerosis (MESA). Eur Heart J 2018;39:2401-8.

33 Pletcher MJ, Tice JA, Pignone M, et al. What does my patient's coronary artery calcium score mean? combining information from the coronary artery calcium score with information from conventional risk factors to estimate coronary heart disease risk. BMC Med 2004;2:31.

34 McClelland RL, Jorgensen NW, Budoff M, et al. 10-Year coronary heart disease risk prediction using coronary artery calcium and traditional risk factors: derivation in the MESA (multi-ethnic study of atherosclerosis) with validation in the HNR (heinz Nixdorf recall) study and the DHS (Dallas heart study). J Am Coll Cardiol 2015;66:1643-53.

35 Ru Zhao Y, Xie X, de Koning $\mathrm{HJ}$, et al. Nelson lung cancer screening study. Cancer Imaging 2011;11 Spec No A:S79-S84.

36 Pedersen JH, Ashraf H, Dirksen A, et al. The Danish randomized lung cancer CT screening trial--overall design and results of the prevalence round. J Thorac Oncol 2009;4:608-14.

37 Sverzellati N, Silva M, Calareso G, et al. Low-Dose computed tomography for lung cancer screening: comparison of performance between annual and biennial screen. Eur Radiol 2016:26:3821-9.

38 Balata H, Blandin Knight S, Barber P, et al. Targeted lung cancer screening selects individuals at high risk of cardiovascular disease. Lung Cancer 2018;124:148-53.

39 Ueda P, Lung TW-C, Lu Y, et al. Treatment gaps and potential cardiovascular risk reduction from expanded statin use in the US and England. PLoS One 2018;13:e0190688

40 Robson J, Dostal I, Sheikh A, et al. The NHS health check in England: an evaluation of the first 4 years. BMJ Open 2016:6:e008840.

41 Mamudu HM, Paul TK, Veeranki SP, et al. The effects of coronary artery calcium screening on behavioral modification, risk perception, and medication adherence among asymptomatic adults: a systematic review. Atherosclerosis 2014;236:338-50.

42 Marshall IJ, Wolfe CDA, McKevitt C. Lay perspectives on hypertension and drug adherence: systematic review of qualitative research. BMJ 2012;345:e3953.

43 Conn VS, Ruppar TM, Chase J-AD, et al. Interventions to improve medication adherence in hypertensive patients: systematic review and meta-analysis. Curr Hypertens Rep 2015;17:94.

44 Kovalchik SA, Tammemagi M, Berg CD, et al. Targeting of low-dose CT screening according to the risk of lung-cancer death. N Engl J Med 2013;369:245-54.

45 Ten Haaf K, Tammemägi MC, Bondy SJ, et al. Performance and cost-effectiveness of computed tomography lung cancer screening scenarios in a population-based setting: a Microsimulation modeling analysis in Ontario, Canada. PLoS Med 2017; 14:e1002225 